\title{
REFERENCES
}

1. I. S. Cohen, Commutative rings with restricted minimum conditions, Duke Math. J. 17 (1950), 27-41.

2. S. MacLane and O. F. G. Schilling, Infinite number fields with Noether ideal theories, Amer. J. Math. 61 (1939), 771-782.

3. N. Nakano, Idealtheorie in einem speziellen unendlichen algebraischen Zahlkorper, J. Sci. Hiroshima Univ. 16(1953), 425-439.

4. O. Zariski and P. Samuel, Commutative algebra. I, Van Nostrand, Princeton, N. J., 1953.

5. —-, Commutative algebra. II, Van Nostrand, Princeton, N. J., 1960.

Louisiana State University and

UNIVERSITY OF WISCONSIN

\section{A NOTE ON PERTURBATION THEORY FOR SEMI-GROUPS OF OPERATORS}

\section{A. OLUBUMMO}

1. Introduction. Let $X$ be a Banach lattice and $\Sigma=\{T(\xi) ; \xi \geqq 0\}$ a family of bounded operators on $X$ satisfying

(i) $T\left(\xi_{1}+\xi_{2}\right)=T\left(\xi_{1}\right) T\left(\xi_{2}\right), T(0)=I, 0 \leqq \xi_{1}, \xi_{2}<\infty$;

(ii) $x \geqq 0$ implies that $T(\xi) x \geqq 0$ for each $\xi \geqq 0$;

(iii) $\|T(\xi)\| \leqq 1$ for $\xi \geqq 0$;

(iv) $\lim _{\xi \downarrow 0} T(\xi) x=x$ for each $x \in X$.

We shall refer to $\Sigma$ as a strongly continuous semi-group of positive contraction operators.

Lumer [2] has shown that for a complex (real) normed linear space $X$, there exists at least one complex-valued (real-valued) function $[x, y]$ called a semi-inner-product defined on $X \times X$ having the following properties:

$$
\begin{aligned}
{[x+y, z] } & =[x, z]+[y, z], \\
{[\lambda x, z] } & =\lambda[x, z], \\
{[x, x] } & =\|x\|^{2} \\
|[x, z]| & \leqq\|x\|\|z\| .
\end{aligned}
$$

If $X$ is a Banach lattice, then Phillips [4] has shown that there exists a semi-inner-product with the further properties:

Received by the editors April 15, 1963. 
$\mathrm{P}$ (a) if $x \geqq 0$, then $[y, x] \geqq 0$ for all $y \geqq 0$,

$\mathrm{P}(\mathrm{b})\left[x, x^{+}\right]=\left\|x^{+}\right\|^{2}$.

Definition. An operator $A$ on a Banach lattice $X$ is called dispersive if $\left[A x, x^{+}\right] \leqq 0, x \in \mathbb{D}(A)$.

In terms of this concept, Phillips has proved the following result [4, Theorem 2.1]:

TheOREM A. A necessary and sufficient condition for a linear operator $A$ with dense domain to generate a strongly continuous semi-group of positive contraction operators on a Banach lattice $X$ is that $A$ be dispersive with $R(I-A)=X$.

Suppose now that $X$ is an abstract $(L)$-space in the sense of $\mathrm{Kaku}$ tani [1]. Then a positive linear functional $(e, \cdot)$ is defined on $X$ by taking

$$
(e, x)=\left\|x^{+}\right\|-\|x-\| .
$$

Reuter [6] and Miyadera [3] have studied the problem of a strongly continuous semi-group $\Sigma^{\prime}=\left\{T^{\prime}(\xi), \xi \geqq 0\right\}$ of positive contraction operators on an abstract $(L)$-space $X$ dominating another such semi-group $\Sigma=\{T(\xi), \xi \geqq 0\}$ in the sense that

$$
T^{\prime}(\xi) x \geqq T(\xi) x \text { for each } x \geqq 0 \text { and } \xi \geqq 0 .
$$

The following result is due to Miyadera [3]:

Theorem B. Let $A$ generate a strongly continuous semi-group $\Sigma$ of positive contraction operators on an abstract $(L)$-space $X$ and let $B$ be a linear operator with domain $D(B) \supset D(A)$. Then $A^{\prime}=A+B$ will generate a strongly continuous semi-group $\Sigma^{\prime}$ of positive contraction operators dominating $\Sigma$ if and only if

(i) $B x \geqq 0$ for $x \geqq 0$ in $D(A)$,

(ii) $(e, B x) \leqq-(e, A x)$ for $x \geqq 0$ in $D(A)$,

(iii) any one of the following:

(a) $R(I-B R(\lambda ; A))=X$ for each $\lambda>0$,

(b) $\sum_{n=0}^{\infty}\left\|[B R(\lambda ; A)]^{n} y\right\|<\infty$ for each $\lambda>0$ and $y \geqq 0$.

The object of this note is to prove an analogue of Theorem $B$ when $X$ is a Banach lattice. It is to be noted that in Theorem B as in our Theorem 1, it is not assumed that the perturbing operator is bounded. Our proof is modelled on Miyadera's proof of Theorem B. In $\S 3$, we consider the case in which the perturbing operator is bounded.

I should like to take this opportunity to thank Professor Ralph Phillips for introducing me to the subject of dispersive operators and for his helpful comments and encouragement in the preparation of this paper. 
2. Unbounded perturbations. The following lemma will be required.

Lemma. Let $A$ be a dispersive operator with domain dense in a Banach lattice $X$ and $\delta$ a real number. If $R(\lambda I-A)=X$ for every $\lambda>\delta$, then $A$ generates a strongly continuous semi-group of positive contraction operators.

This is proved by Phillips in the process of proving Theorem A. The following is our main result:

THEOREM 1. Let $A$ generate a strongly continuous semi-group $\Sigma$ of positive contraction operators on a Banach lattice $X$ and let $B$ be $a$ linear operator with domain $D(B) \supset D(A)$. Then $A^{\prime}=A+B$ generates a strongly continuous semi-group $\Sigma^{\prime}$, of positive contraction operators dominating $\Sigma$ if and only if

(1) $B x \geqq 0$ for $x \geqq 0$ in $D(A)$,

(2) $\left[B x, x^{+}\right] \leqq-\left[A x, x^{+}\right]$for $x \in \mathcal{D}(A)$,

(3) $\mathbb{R}(I-B R(\lambda ; A))=X$ for each $\lambda>0$.

Proof. Let $\{T(\xi), \xi \geqq 0\}$ and $\left\{T^{\prime}(\xi), \xi \geqq 0\right\}$ denote the operators in the semi-groups $\Sigma$ and $\Sigma^{\prime}$, respectively. Then the necessity of (1) follows from

$$
(A+B) x=\lim _{\xi \downarrow 0} \frac{1}{\xi}\left[T^{\prime}(\xi)-I\right] x \geqq \lim _{\xi \downarrow 0} \frac{1}{\xi}[T(\xi)-I] x=A x
$$

for $x \geqq 0$ in $D(A)$ and that of (2) follows from Theorem A. To prove the necessity of (3), we note that

$$
\left(\lambda I-A^{\prime}\right) R(\lambda ; A) x=(I-B R(\lambda ; A)) x, \quad x \in X ;
$$

thus

$$
\begin{aligned}
(I-B R(\lambda ; A))[X] & =\left(\lambda I-A^{\prime}\right) R(\lambda ; A)[X]=\left(\lambda I-A^{\prime}\right)[D(A)] \\
& =\left(\lambda I-A^{\prime}\right)\left[D\left(A^{\prime}\right)\right]=X .
\end{aligned}
$$

Suppose now that (1), (2) and (3) hold. We first show that the inverse $(I-B R(\lambda ; A))^{-1}$ exists for each $\lambda>0$. Let $(I-B R(\lambda ; A)) x=0$ for some $x \in X$. Since $B R(\lambda ; A)$ is a positive operator,

$$
|x|=|B R(\lambda ; A) x| \leqq B R(\lambda ; A)|x|
$$

and since $A+B$ is dispersive,

$$
[(B+A) R(\lambda ; A)|x|, R(\lambda ; A)|x|] \leqq 0 .
$$

Thus 


$$
\begin{aligned}
& {[B R(\lambda ; A)|x|, R(\lambda ; A)|x|]} \\
& \quad \leqq-[A R(\lambda ; A)|x|, R(\lambda ; A)|x|] \\
& \quad \leqq-[\lambda R(\lambda ; A)|x|-|x|, R(\lambda ; A)|x|] \\
& \quad \quad(\text { since } A R(\lambda ; A)|x|=\lambda R(\lambda ; A)|x|-|x|) \\
& \quad \leqq[|x|, R(\lambda ; A)|x|]-[\lambda R(\lambda ; A)|x|, R(\lambda ; A)|x|] \\
& \quad \leqq[B R(\lambda ; A)|x|, R(\lambda ; A)|x|]-\lambda[R(\lambda ; A)|x|, R(\lambda ; A)|x|],
\end{aligned}
$$

where the last inequality holds by virtue of $\mathrm{P}(\mathrm{a})$. Then $\lambda\|R(\lambda ; A)|x|\|$ $=0$ and therefore $\lambda R(\lambda ; A)|x|=0$, whence $|x|=(\lambda I-A) R(\lambda ; A)|x|$ $=0$ and $(I-B R(\lambda ; A))^{-1}$ exists for $\lambda>0$. Further, by (3), the domain of $(I-B R(\lambda ; A))^{-1}$ is $X$.

We now set $R\left(\lambda ; A^{\prime}\right)=R(\lambda ; A)(I-B R(\lambda ; A))^{-1}$. Then $\left(\lambda I-A^{\prime}\right) R\left(\lambda ; A^{\prime}\right)=(I-B R(\lambda ; A))(I-B R(\lambda ; A))^{-1}=I$. Also the range of $R\left(\lambda ; A^{\prime}\right)$ is precisely $D(A)$ since the range of $(I-B R(\lambda ; A))^{-1}$ is $X$. Thus, given $x \in D(A)$, there exists $y \in X$ such that $x=R\left(\lambda ; A^{\prime}\right) y$. Hence $R\left(\lambda ; A^{\prime}\right)\left(\lambda I-A^{\prime}\right) x=R\left(\lambda ; A^{\prime}\right)\left(\lambda I-A^{\prime}\right) R\left(\lambda ; A^{\prime}\right) y=R\left(\lambda ; A^{\prime}\right) y$ $=x$. Further, by (2), $\left[A^{\prime} x, x^{+}\right] \leqq 0$ for $x \in D(A)$. Thus $A^{\prime}$ satisfies the conditions of Lemma 1 and hence generates a strongly continuous semi-group $\Sigma^{\prime}=\left\{T^{\prime}(\xi), \xi \geqq 0\right\}$ of positive contraction operators. Moreover, since $R(\lambda ; A)$ and $B$ are both positive operators, it follows that $R\left(\lambda ; A^{\prime}\right) x \geqq R(\lambda ; A) x$ for $x \geqq 0$ and $\lambda>0$. The formula

$$
T(\xi) x=\lim _{\lambda \rightarrow \infty}\left\{\exp (-\lambda \xi) \sum_{n=0}^{\infty}\left(\lambda^{2} \xi\right)^{n}[R(\lambda ; A)]^{n} / n !\right\} x
$$

now shows that $T^{\prime}(\xi) x \geqq T(\xi) x$ for each $\xi \geqq 0$ and $x \geqq 0$. This concludes the proof.

3. Bounded perturbations. We now specialize to the case in which the perturbing operator is bounded.

TheOREM 2. Let $A$ generate a strongly continuous semi-group $\Sigma$ of positive contraction operators on a Banach lattice $X$ and let $B$ be a bounded linear operator with domain $D(B) \supset D(A)$. Then $A^{\prime}=A+B$ generates $a$ strongly continuous semi-group $\Sigma^{\prime}$ of positive contraction operators dominating $\Sigma$ if and only if

(1) $B x \geqq 0$ for $x \geqq 0$ in $\mathfrak{D}(A)$,

(2) $A^{\prime}=A+B$ is dispersive.

Proof. The necessity part follows as in Theorem 1. Suppose now that (1) and (2) hold. Since $A$ generates a strongly continuous semigroup of contraction operators, there exist real numbers $M>0$ and $\boldsymbol{\gamma}$ such that 


$$
\left\|[R(\lambda ; A)]^{n}\right\| \leqq M(\lambda-\gamma)^{-n} \quad \text { for } \lambda>\gamma .
$$

(For a proof of this, see [5, Theorem 2.1].) Set $\gamma_{1}=\gamma+M\|B\|$; then for $\lambda>\gamma_{1}$, we have

$$
\|B R(\lambda ; A)\| \leqq\|B\|\|R(\lambda ; A)\| \leqq\|B\| M /(\lambda-\gamma)<1 .
$$

Then by [5, Lemma 3.1], $R\left(\lambda ; A^{\prime}\right)$ exists and

$$
R\left(\lambda ; A^{\prime}\right)=\sum_{n=0}^{\infty} R(\lambda ; A)[B R(\lambda ; A)]^{n}=R(\lambda ; A)[I-B R(\lambda ; A)]^{-1},
$$

if $\lambda>\gamma_{1}$, the series converging absolutely. It follows as before that $R\left(\lambda ; A^{\prime}\right)$ is both a left and a right inverse of $\lambda I-A^{\prime}$. Thus again in view of (2), $A^{\prime}$ satisfies the conditions of Lemma 1 and hence generates a strongly continuous semi-group $\Sigma^{\prime}$ of positive contraction operators. The proof is now completed as in Theorem 1.

\section{REFERENCES}

1. S. Kakutani, Concrete representations of abstract $(L)$-spaces and the mean ergodic theorem, Ann. of Math. (2) 42 (1942), 523-537.

2. G. Lumer, Semi-inner product spaces, Trans. Amer. Math. Soc. 100 (1961), 29-43.

3. I. Miyadera, $A$ note on contraction semi-groups of operators, Tôhoku Math. J. (2) 11 (1959), 98-105.

4. R. S. Phillips, Semi-groups of positive contraction operators, Czechoslovak. Math. J. 12(87) (1962), 294-313.

5. - Perturbation theory for semi-groups of linear operators, Trans. Amer. Math. Soc. 74 (1953), 199-221.

6. G. E. H. Reuter, A note on contraction semi-groups, Math. Scand. 3 (1955), 275-280.

UNIVERSITy OF IBADAN, Nigeria 